Objective To evaluate the effect of HIV infection on atherosclerosis in asymptomatic patients.

Subjects and methods Study design: observational, prospective case-control study, including 124 consecutive male and female HIVinfected patients, older than 18 year (HIV-group). Results were compared with 130 healthy volunteers of same gender and age (Control-group).

Study protocol: Clinical evaluation followed by ultrasound exam of carotids for carotid intimal-medial thickness (CIMT) measurement. The presence of atherosclerosis plaques was recorded.

Statistical analysis: Chi-square test and linear regression analysis. Significance level: $p<0.05$.

Result Age (HIV-group:43.87 \pm 11.31 vs Control-group: $42.9 \pm$ $11.54, p=0.324)$ was similar in both groups. There was $52.00 \%$ male in HIV-group and $54.00 \%$ in Control group. Atherosclerotic plaque was identified in $30.64 \%$ of the patients HIV and in $3.84 \%$ of the control ( $p<0.001$ ). Surprisingly, $22 \%$ of HIV-infected patients, classified at low risk score Freminghan, presented plaques in the carotid arteries.

For controls, there was an increase of $0.068 \mathrm{~mm}$ in the CIMT for each one-year increase in age (OR: 1.068; CI95\%: 1.03-1.107; $\mathrm{p}<0.001)$. The presence of HIV increased this increment for tenfold (OR: 10.7; CI95\%: 3.58-31.76; $\mathrm{p}<0.001$ ). There was an interaction between age and HIV-infection to increase CIMT ( $p<0.001)$.

Conclusions Our results indicated that: 1 - patients with HIV are at higher risk for atherosclerosis in the carotid artery than control individuals. 2- The effect of age on risk for atherosclerosis occurs in both groups, however it is more remarkable in HIV-infected patients.

\section{P5.109 KNOWLEDGE AND UPTAKE OF HIV INTERVENTION AND PREVENTION SERVICES IN MANICALAND, ZIMBABWE: CURRENT DETERMINANTS OF COVERAGE AND PROGRESS OVER TIME}

doi:10.1136/sextrans-2013-051184.1153

'N Schur, ${ }^{1,2} \mathrm{C}$ Nyamukapa, 1,2S Gregson. 'Department of Infectious Disease Epidemiology, Imperial College London, London, UK; ${ }^{2}$ Biomedical Research and Training Institute, Harare, Zimbabwe

Background Zimbabwe has experienced one of the world's most severe HIV crises since the first reported case of AIDS in the country in 1985. Over the last decade, the Zimbabwe Government has implemented a range of services to prevent new infections and to support people living with HIV, e.g. HIV testing and counselling (HTC), anti-retroviral treatment (ART), prevention of mother-tochild transmission (PMTCT), and male circumcision (MC).

We describe trends and patterns in knowledge and uptake of these services, investigate individual-level factors associated with greater levels of coverage, and analyse shortfalls in order to inform future policies on the scale-up of HIV services.

Methods Data from the latest round of a large $(n \approx 12,000)$ population-based cohort study conducted between 2009 and 2011 in eastern Zimbabwe will be used in logistic regression models to analyse associations between demographic, socio-economic, behavioural and other factors, and knowledge and uptake of services. Temporal trends in uptake of services will be measured using data from earlier rounds of the study.

Results HIV prevalence in the general population of Manicaland province remained comparably high $(15.1 \%$; $95 \%$ confidence interval: $14.5 \%-15.7 \%)$. Preliminary univariate results suggest that knowledge of HIV services was high for HTC (82.3\%; 91.6\%-82.9\%) and PMTCT $(93.0 \% ; 92.0 \%-93.9 \%)$. Knowledge of ART was low overall (40.1\%; 39.3\%-40.9\%) but high in self-reported HIV-positives $(88.9 \% ; 86.8 \%-90.7 \%)$. Overall uptake of services in the last three years was moderate for HTC (44.0\%; 43.2\%-44.8\%) and PMTCT
(65.2\%; $57.1 \%-72.6 \%)$. Current uptake of ART in self-reported HIV positives (79.2\%; 76.3\%-81.8\%) almost reached universal coverage, which is defined at $85 \%$ in Zimbabwe, but only about every second lab-confirmed HIV positive person in the study population was on treatment $(52.2 \% ; 49.2 \%-55.1 \%)$. MC uptake in the male population remained low $(8.2 \% ; 7.5 \%-8.9 \%)$. Knowledge and uptake of services varied by sex and age and may also differ by other factors.

\section{P5.110 MULTIPROFESSIONAL APPROACH FOR THE DEVELOPMENT OF THE NATIONAL STIS MANAGEMENT GUIDELINES IN LITHUANIA}

doi:10.1136/sextrans-2013-051184.1154

'V Kucinskiene, 'S Valiukeviciene, 'A Vitkauskiene, ${ }^{2} \mathrm{M}$ Domeika, Eastern European Network for SexualReproductive Health. 'Department of Skin and Venereal Diseases, Medical Academy, Lithuanian University of Health Sciences, Kaunas, Lithuania; ${ }^{2}$ Department of Control and Prevention of Communicable Diseases Uppsala County Council, Uppsala, Sweden

Background During many decades in the majority of Eastern European Countries, persons with lower genital tract infections were treated exceptionally by the veneologist. Today dermatovenereologist sees less than $40 \%$ of these patients. The rest of the patients is treated and managed by gynaecologists, general physicians, urologists etc. Therefore in developing STI patient management approaches a multiprofessional approach is crucial.

Methods Recently, collaborating effort to prepare STI patient management guidelines in Lithuania was undertaken. Dermatovenereologists, gynaecologists, urologists, microbiologists, general practitioners and other STI patients managing specialists for the first time gathered together. IUSTI, WHO, CDC and EE SRHR guidelines were used.

Results Collaborating project resulted in development of evidencebased STI patient management guidelines, containing the information about the: (i) who should be tested; (ii) what methods are to be used; (iii) recommended treatment regiments; (iv) partner management strategies; (v) follow up approaches; (vi) case reporting and (vi) counselling. Syndromic approach for the management of urethritis and cervicitis as well as complications has also been described. Over 1200 copies of the guidelines have been disseminated to medical professionals and made available for medical students. The electronic version of the document has been presented at the website www. kaunoklinikos. It and can be accessed to any specialist and medical student with no charge. During the preparation of the guidelines the main discrepancies with the international practise identified and eliminated, as e.g. use of serology for diagnosis of chlamydial infection, the use microscopy and not culture, no NAATS for the diagnosis of gonorrhoea, treatment of early syphilis by short acting penicillin, preventive treatment of syphilis for pregnant women, etc. Conclusion STI management guidelines became an excellent example of the multiprofessional collaboration in Lithuania, bringing international evidence-based approaches across the professional barriers of all medical professions, involved in management of STIpatients.

\section{P.06 - Health Policy and Programme Sciences Track}

P6.001 FACING THE FUTURE TOGETHER: EMBODYING
APPRECIATIVE INQUIRY AND STAFF ENGAGEMENT IN
PLANNING CHANGE IN SEXUAL HEALTH SERVICES IN
GLASGOW, SCOTLAND, UK

doi:10.1136/sextrans-2013-051184.1155

${ }^{1,2} \mathbf{R}$ llett. 'Sandyford Sexual Health Services, Glasgow, UK; ${ }^{2}$ University of Glasgow, Glasgow, UK 
Background After 12 years of activity the integrated sexual health service in Glasgow, Scotland begun to plan for the future. The service's evolution from previously separate units improved access to sexual health care, however, maintaining and growing innovative services had become challenging, with restricted resources and staffing, and complex client demand. The service now has 20 sites, 250 clinical, support and management staff and sees 100, 000 clients annually.

When its manager returned from another role, the service started to consider its future delivery model. This paper sets out the methodology based around appreciative inquiry and staff engagement, and includes feedback from staff about its impact on them and the organisation.

Methods The process began in June 2012 with a Planning Day for senior staff which agreed a timetable, identified staff and others to involve; scoped essential areas such as public engagement, consultation, communication, and evidence like population projections and service data, and agreed a shared vision. Personal narratives and goals were shared to shape the vision and to acknowledge the past. A multi-disciplinary Steering Group was then established to participate in planning and decision-making, with a Staff Council arising through staff interest - both now with significant roles. Regular staff communications and large engagement events have also helped strengthen plans and organisational structures.

Results The service's future vision for developing sexual health services acknowledges its history and proposes clear objectives that re-iterate a commitment to addressing inequalities and to proportionate universalism. This has been achieved in an inclusive manner that has empowered staff across the organisation and included their views and ideas.

Summary This paper describes a systematic approach to engaging with a large and diverse staff group through appreciative inquiry and other methods to improve the sexual health services in the Glasgow area of Scotland.

\section{P6.002 EFFICACY OF CHLAMYDIA CONTROL PROGRAMMIS: OPTIMAL COMBINATIONS OF PARTNER NOTIFICATION AND SCREENING IN A PAIR APPROXIMATION MODEL}

doi:10.1136/sextrans-2013-051184.1156

1J Clarke, ${ }^{2} \mathrm{~J}$ K White, ${ }^{2} \mathbf{K}$ M Turner. 'University of Bath, Bath, UK; ${ }^{2}$ University of Bristol, Bristol, UK

Background Chlamydia control in England is based on management of positives and their partners (partner notification) and on identifying asymptomatic infections in the community (screening).

Aims To describe the relative contribution of screening and partner notification to control of chlamydia at different stages in the epidemic and evaluate optimal resource allocation to each control.

Methods Using pair approximation equations we investigate the efficacy of control programmes for chlamydia on short time scales that are relevant to policy makers. We estimate prevalence, incidence, and positivity in those screened and in their partners. We combine these measures with a costing tool to estimate the economic impact of different public health strategies.

Results Increasing screening coverage significantly increases the annual programme costs whereas an increase in tracing efficiency initially increases annual costs but over time reduces costs below baseline, with tracing accounting for around $10 \%$ of intervention costs. We found that partner positivity is insensitive to changes in population prevalence due to screening, remaining at around $33 \%$.

Conclusions Maintaining efficacy of partner notification is costeffective at all stages of the epidemic (stable/declining prevalence) but becomes increasingly cost-effective as control measures reduce the population prevalence.

\section{P6.003 GEOGRAPHIC MAPPING OF FEMALE SEX WORKERS AND VENUE PROFILING IN URBAN AND RURAL DISTRICTS OF ZIGONG, SICHUAN, CHINA}

doi:10.1136/sextrans-2013-051184.1157

'J F Blanchard, ${ }^{2} \mathrm{~J}$ Zhang, ${ }^{2} \mathrm{H}$ Zhou, ${ }^{2} Y$ Yang, ${ }^{3} Y \mathrm{Xie},{ }^{3} \mathrm{O} \mathrm{Li},{ }^{1} \mathrm{~F}$ Emmanuel, ${ }^{2} \mathrm{X} \mathrm{Ma},{ }^{1} \mathrm{R}$ Lorway, 'B Yu. 'University of Manitoba, Winnipeg, MB, Canada; ${ }^{2}$ Sichuan University, Chengdu, China; ${ }^{3}$ Zigong Center for Disease Prevention and Control, Zigong, China

Introduction The purpose of this study is to geographically enumerate the population size of female sex workers (FSWs) and venue profiling of key venues, and to reveal the key elements which are related to the high risk sexual behaviours among FSWs in urban and rural areas of Zigong city in Sichuan, China.

Methods Geographic mapping data were collected through systematically identifying hidden key venues in the rural and urban districts of Zigong. Venue profiling data were collected by interviewing key informants (KI) about the details of sex work operation, such as type of venue, duration of operation, operation days and time, peak days and time, services provided at each venue, number of clients on an average day and a peak day. To avoid social desirability bias of face-to-face interview, Polling Booth Survey was used to gather high risk sexual behaviours among FSWs $(\mathrm{N}=60)$.

Results A total of 324 key venues were mapped in Zigong. The key venues are massage parlours (108), teahouses (74) and small hotels (45), which accounting for $33.3 \%, 22.8 \%$ and $13.9 \%$ of total venues mapped. 112 venue KIs were interviewed and confirmed a total of 378 FSWs working in those 112 venues. The average number of FSWs per venue is 4 . The age of the majority (80.4\%) of FSWs was around 20 to 40 years old. The total estimated number of FSWs in Zigong is 1296 . The sexual behaviours and operation patterns of key venues in urban and rural areas are different. Not consistent condom use, STI symptoms, and drug use are some typical high risk behaviours.

Discussion and Conclusion Sex work industry is emerged in general social life in urban and rural China. The scope and operation of sex industry pose a special challenge to public health intervention programmes

\section{P6.004 THE PREVALENCE OF SEXUALIY TRANSMITTED INFECTIONS AMONG CLIENTS OF FEMALE SEX WORKERS IN KARNATAKA, SOUTH INDIA: AN ANALYSIS BY PLACES OF SOLICITATION OF SEX WORKERS}

doi:10.1136/sextrans-2013-051184.1158

${ }^{1} \mathbf{S}$ Y Shaw, ${ }^{2} \mathrm{P}$ Bhattarcharjee, ${ }^{2} \mathrm{~S}$ Isac, ${ }^{3} \mathrm{~K} N$ Deering, ${ }^{2} \mathrm{~B} M$ Ramesh, ${ }^{2} \mathrm{R}$ Washington, ${ }^{1} S$ Moses, ' J F Blanchard. 'University of Manitoba, Winnipeg, MB, Canada; ${ }^{2}$ Karnataka Health Promotion Trust, Bangalore, India; ${ }^{3}$ University of British Columbia, Vancouver, $B C$, Canada

Background Clients of female sex workers (FSWs) are an important bridging population for HIV/STI transmission. Designing client-specific intervention programming faces several challenges, as clients do not comprise one single identifiable group. This study sought to describe HIV/STI prevalence by places of solicitation, among clients in south India.

Methods Data were from two rounds of cross-sectional biological and behavioural surveys of FSW clients from Karnataka State, India. Where available, the prevalence of HIV, active syphilis, herpes simplex virus type 2 (HSV-2), chlamydia (CT) and gonorrhoea (GC) was examined. Separately for each survey round and infection, multivariable logistic regression models were used to examine differences in infection prevalence by solicitation site, adjusted for clients' district. Solicitation sites were categorised as public place, brothel, home and lodge. Sampling weights and survey methods were utilised in regression models. 